Relations industrielles

Industrial Relations

\title{
The Forest Ranger, a study in administrative behavior, by Herbert Kaufman, Johns Hopkins Press, Homewood, Baltimore 18, Maryland, pp. 259.
}

\section{Émile Gosselin}

Volume 17, numéro 3, juillet 1962

URI : https://id.erudit.org/iderudit/1021586ar

DOI : https://doi.org/10.7202/1021586ar

Aller au sommaire du numéro

Éditeur(s)

Département des relations industrielles de l’Université Laval

ISSN

0034-379X (imprimé)

1703-8138 (numérique)

Découvrir la revue

Citer ce compte rendu

Gosselin, É. (1962). Compte rendu de [The Forest Ranger, a study in administrative behavior, by Herbert Kaufman, Johns Hopkins Press, Homewood, Baltimore 18, Maryland, pp. 259.] Relations industrielles / Industrial Relations, 17(3), 354-355. https://doi.org/10.7202/1021586ar

Tous droits réservés (C Département des relations industrielles de l’Université Laval, 1962
Ce document est protégé par la loi sur le droit d'auteur. L'utilisation des services d’Érudit (y compris la reproduction) est assujettie à sa politique d'utilisation que vous pouvez consulter en ligne.

https://apropos.erudit.org/fr/usagers/politique-dutilisation/ 
Puis vient, au cinquième chapitre, une étude des relations industrielles au niveau local, étude qui couvre en fait les principales phases de l'administration des relations journalières et des problèmes auxquels elles donnent lieu au plan de l'usine, de l'unité de production. On y traite, entre autres choses, des dimensions nouvelles des unités de production, de leur évolution technologique, des problèmes d'apprentissage, de formation du personnel, de recrutement, de communications, etc. Le sixième chapitre s'attaque aux aspects sociaux de l'emploi industriel. La question des bénéfices marginaux (fringe benefits) et des autres mesures de sécurité sociale y est étudiée particulièrement du point de vue de leur coût, de leurs relations avec les salaires, et de leur administration dans le contexte changeant dont l'étude forme la trame de tout le volume.

Les trois derniers chapitres sont consacrés aux cadres juridiques dans lesquels fonctionnent les relations du travail en Grande-Bretagne présentement, ainsi qu'aux développements en perspectives dans l'appareil légal conditionnant ces relations (Chapitre 7); puis ils traitent des rapports entre la politique économique du pays et l'action syndicale (Chapitre 8), pour conclure avec une étude des politiques gouvernementales et du rôle rénové que doit y assumer le Minitsère du Travail par la voie de ses différentes agences en matière de direction, de conciliation et conseil aux parties en cause.

Ce qui frappe le lecteur nord-américain en parcourant cet ouvrage, c'est en dépit des distances géographiques et institutionnelles séparant l'Angleterre de notre continent, la similitude très grande des problèmes de relations industrielles confrontant la Grande-Bretagne présentement avec ceux auxquels Américains et Canadiens ont à faire face de ce côté-ci de l'Atlantique. La question du danger inflationnaire, celle des structures syndicales quelque peu sclérosées, celles encore des institutions entourant la détermination des salaires, enfin celles de l'automation, des transformations de la maind'oeuvre et du recrutement syndical, sont des thèmes bien connus ici parmi les questions urgentes auxquelles répondre et ce sont encore ces mêmes questions que nous retrouvons en substance au coeur des préoccupations des élites industrielles britanniques.
Ne serait-ce que pour y découvrir cette preuve supplémentaire que l'étude des relations du travail porte de plus en plus sur des données similaires et universelles, un tel volume vaut d'être consulté. Cette remarque, il va sans dire, ne diminue en rien l'excellence des études qui y sont présentées et qui, en soi, comportent un intérêt capital pour toute personne intéressée par les choses du travail.

Jean-Réal Cardin

The Forest Ranger, a study in administrative behavior, by Herbert Kaufman, Johns Hopkins Press, Homewood, Baltimore 18, Maryland, pp. 259.

Herfbert Kaufman a déjà publié de nombreux articles sur l'administration et le gouvernement. Son plus récent ouvrage que nous présentons au lecteur apporte des points de vue qui nous sortent des sentiers battus et des clichés de l'administration. Aussi les administrateurs de profession ainsi que les spécialistes de la politique, de l'administration publique et de la rationalisation du travail devraient le consulter avec grand intérêt. Les forestiers devraient également y trouver leur profit car ils souscrivent sans doute à ce que souligne l'auteur :

$\ll .$. lands of our nation are too extensive and too important for us to tolerate administration or management at less than the full economic potential. »

Le lecteur qui se croit renseigné en méthodes forestières sera déçu de ne pas $y$ trouver de nouvelles techniques en vue d'une meilleure utilisation de la scie à chaînette ou du bélier mécanique. Ce qui intéresse le chercheur, c'est le problème de la coordination entre les responsables de l'établissement des politiques forestières et les officiers subalternes. Où et comment les responsables d'une large organisation peuvent-ils atteindre leurs objectifs?

Dans son ouvrage «Administrative Behavior 》 (N.York-Macmillan Co., 1947, pp. 2-3) développe la thèse que dans une organisation «The actual physcial task of carrying out an organization's objectives falls to the persons at the lowest level of the administrative hierarchy ». Aussi, l'employé dans la ligne d'exécution doit devenir le centre de l'attention car le succès de l'entreprise 
dépendra de son comportement. L'on comprend mieux la structure et la fonction d'une organisation si l'on analyse de quelle façon les décisions et le comportement de tels employés sont influencés au sein de l'organisation.

L'étude porte sur le service de protection des forêts, une division du Département de l'Agriculture des E.-U. Après avoir fait une longue analyse de l'ampleur et de la complexité des divers services forestiers, l'auteur étudie les problèmes majeurs qui se posent: problèmes de communication dus à la distance, à l'interprétation des lois et règlement., relations avec le public, sélection des officiers, déconcentration des services. Si de tels problèmes n'étaient pas surveillés de près, l'ensemble de l'organisation aurait vite tendance à se fragmenter, et elle cesserait d'agir comme un tout fonctionnel et bien intégré. Or l'auteur démontre comment les divers facteurs de désintégration sont maintenus sous contrôle et les politiques mises en oeuvre pour faire du service une organisation travaillant de façon harmonieuse et bien coordonnée. Il analyse de quelle façon les politiques sont élaborées, quelles soient d'ordre financier ou purement administratif, et comment l'on détecte et corrige toute déviation aux normes, une fois qu'elles auront été établies. Enfin, l'auteur démontre qu'il n'est pas suffisant pour les membres d'une organisation de posséder uniquement une compétence purement technique, mais ils doivent être capables de faire équipe et d'exécuter spontanément les programmes selon les politiques et les directives.

\section{EMme Gosselin}

Dictionnaire économique et social, par Thomas Suavet. Coll. «Initiation économique ». Economie et Humanisme. Les Editions ouvrières, 12, avenue Soeur-Rosalie, Paris (13), 1962, $455 \mathrm{pp}$.

Comme nous en avertit l'auteur, cet ouvrage n'est pas destiné aux spécialistes qui veulent accroître leurs connaissances dans leur domaine. Mais il leur permet d'ouvrir leurs horizons et de trouver certains éléments utiles à une meilleure compréhension de l'ensemble du champ économique et social.

L'auteur a réussi à éviter deux extrèmes: présenter un simple compendium de mots choisis dans Larousse ou Quillet, d'une part, et, d'autre part, de faire une encyclopédie. Autour d'environ trois cents mots-clés, il a synthétisé un ensemble de notions théoriques et de données factuelles dont la connaissance est indispensable à tous ceux qui travaillent dans les domaines social, économique ou politique. La plupart des articles sont suivis d'une brève bibliographie. A la fin de l'ouvrage, on trouve un répertoire alphabétique des mots analysés soit dans un article spécial ou dans un autre indiqué par un renvoi.

L'auteur s'est donné beaucoup de peine pour présenter un instrument de travail à la fois bref et abordable. Parce que cet ouvrage a été préparé pour la France, les références aux problèmes concrets sont illustrées soit par l'histoire, la législation et les institutions de ce pays. Si l'auteur avait voulu tenir compte des lecteurs en dehors de la France, il aurait été obligé d'accroître considérablement le format de son dictionnaire et de faire appel à la collaboration de plusieurs étrangers d'expression française. Même si pour cette raison, les lecteurs canadiens ne peuvent tirer autant de bénéfices que les lecteurs français, ils trouveront encore suffisamment avantage à posséder un tel ouvrage et à le consulter. Tout ce que nous pouvons souhaiter, c'est qu'un jour soit publié un supplément pour le Canada et les autres pays d'expression française.

\section{GÉrard Dion}

Studies in Social Power. Dorwin Cartwright, éditeur, Research Center for Group Dynamics, Institute for Social Research, The University of Michigan, Ann Arbor, 1959, pp. 225.

Ce volume représente le résultat de travaux et de recherches qui se sont étendus sur une période de huit années. Chaque chapitre étudie un aspect déterminé du pouvoir dans la société, soit en tant que cause ou encore en tant qu'effet. Par exemple: quels sont les sources ou les déterminants du pouvoir? Sous quelles conditions la possession du pouvoir devient-elle de fait l'exercice du pouvoir! Comment le pouvoir affecte$t$-il les communications entre personnes ? Le pouvoir constitue-t-il une menace pesant sur ceux qui l'exercent! Quels sont les effets de la possession du pouvoir sur 